\title{
INVESTIGAÇÃO SOBRE SINTOMAS DE TRANSTORNO DE ESTRES- SE PÓS-TRAUMÁTICO EM POLICIAIS: UM ESTUDO A PARTIR DO GRUPO DE OPERAÇÕES ESPECIAIS (GOE) DE CACOAL-RO
}

RESEARCH ON SYMPTOMS OF POST-TRAUMATIC STRESS DISORDER IN POLICE: A STUDY FROM CACOAL-RO'S GROUP OF SPECIAL OPERATIONS (GOE)

\section{Cleber Lizardo de Assis', Marilani Sabino da Silva²}

RECEBIDO: 08/06/2017 | ACEITO: 06/11/2018

DOI: $10.5902 / 2317175827578$

\section{RESUMO}

A profissão da polícia militar (GOE) é considerada de risco ao desenvolvimento do Transtorno de Estresse Pós-Traumático pela exposição a eventos traumáticos e catastróficos no âmbito de sua atividade. O objetivo desse estudo foi verificar a existência de sintomas do Transtorno de Estresse Pós-Traumático no grupo de Operações Especiais (GOE) de Cacoal, Rondônia, com uma amostra de 09 (nove) indivíduos. Os dados da pesquisa foram obtidos através da aplicação da escala de PCL-C, composta por 17 itens e dividida em três dimensões: a reexperiência do trauma, a evitação e a hiperestimulação. Pontuando um corte de $100 \%$, descartando a existência do TEPT nos policiais, houve, porém, um índice relevante nas dimensões de hiperestimulação e evitação da escala PCL-C, no qual 08 sujeitos, equivalente a $88,9 \%$, disseram sentir-se em estado de "superalerta", vigilante ou "em guarda". Conclui-se que, mesmo não identificando indícios de TEPT, há prevalência de alguns sintomas desse transtorno em diversos indivíduos e possíveis prejuízos no seu funcionamento psicossocial. Portanto, é de grande relevância o apoio psicológico dentro da corporação, de modo a estimular ações de promoção da saúde mental.

Palavras-chave: Transtorno de Estresse Pós-Traumático, Policiais Militar, Saúde Mental, Trabalho.

1 Mestre em Psicologia (PUCMG), Doutor em Psicologia (USAL-AR); Pós-Doutor em Filosofia (FAJE-MG). E-mail: kebelassis@yahoo.com.br.

2 Graduada em Psicologia, FACIMED - RO 
INVESTIGAÇÃO SOBRE SINTOMAS DE TRANSTORNO DE ESTRESSE PÓS-TRAUMÁTICO EM POLICIAIS:

UM ESTUDO A PARTIR DO GRUPO DE OPERAÇÕES ESPECIAIS (GOE) DE CACOAL-RO

\section{ABSTRACT}

The military police profession (GOE) is considered to be at risk for the development of Post Traumatic Stress Disorder due to exposure to traumatic and catastrophic events within the scope of its activity. The objective of this study was to verify the incidence of Post Traumatic Stress Disorder in the Special Operations group (GOE) of Cacoal RO, with a sample of 09 (nine) subjects. The research data were obtained through the application of the PCL-C scale composed of 17 items divided into three dimensions; Reexperience of trauma, Avoidance and Hyperstimulation. However, there was a relevant index in the dimensions of Hyperestimulation and Avoidance of the PCL-C scale, in which 08 subjects said they felt in a "super-alert" state, Vigilant or "on guard" equivalent to (88.9\%). It is concluded that, even without identifying signs of PTSD, there is a prevalence of some symptoms in large part of the corporation and possible damages in the psychosocial functioning. It is therefore recommended that psychological support within the corporation is of great relevance in order to promote actions to promote mental health

Keywords: Post Traumatic Stress Disorder, Military Police, Mental Health, Work.

\section{Introdução}

O Transtorno de Estresse Pós-Traumático está relacionado a um conjunto de fatores nos quais o sujeito é exposto, seja de forma direta ou indireta, a eventos traumáticos, como ameaça de morte, violência sexual e lesões graves. Os aparecimentos dos sintomas geralmente ocorrem nos primeiros três meses ou até anos após o evento traumático, do qual o sujeito possui memórias e recordações com a mesma intensidade de sofrimento e dor sentidos no momento do evento ocasionando prejuízos significativo na vida do mesmo (DSM5 - American Psychiatry Association, 2014).

Sabendo que as exposições a eventos traumáticos podem ser um fator para desencadear o TEPT (Transtorno de Estresse Pós-Traumático), as funções laborais praticadas pelos policiais da força tática propiciam o desenvolvimento desse quadro e assim acarretam no afastamento do indivíduo de suas funções. Paralelo a isso, aumentam as dificuldades de compreensão devido ao desconhecimento sobre o distúrbio (RIBEIRO, 2015).

O G.O.E (Grupo de Operações Especiais) é uma unidade de recursos especiais e tem por atribuições básicas prestar auxílio, às autoridades policiais e seus agentes, no desempenho das missões de polícia judiciária e no desenvolvimento de atividades de alta complexidade. Essas atividades, possivelmente, corroboram para que os indivíduos se tornem vulneráveis à fatores desencadeadores do TEPT (Transtorno de Estresse Pós-Traumático), devido a realização da atividade com alta exposição marcante e execução de estratégias rápidas para obterem a eficácia nos atendimentos. 


\section{Fundamentação teórica}

\subsection{Transtorno de Estresse Pós-Traumático}

Conforme a 5a edição do DSM-5 2014 (Manual Diagnóstico e Estatística de Transtornos Mentais), o TEPT é um transtorno de ansiedade relacionado a trauma e estressores, tendo o desenvolvimento de sintomas característicos após a exposição a um ou mais eventos traumáticos. Pode ocorrer em qualquer idade a partir do primeiro ano de vida, sendo que os sintomas geralmente se manifestam dentro dos primeiros meses depois do trauma e a ansiedade está presente constantemente (DSM-5, 2014). Na CID-10 (OMS, 1993), o TEPT faz parte dos quadros associados às reações ao estresse e aos transtornos de ajustamento (F43). Tendo como sintomas a revivência, sensação de entorpecimento e embotamento afetivo, evitação, anedonia e hiperexcitabilidade fisiológica, os quais são sintomas padronizados em relação ao público vulnerável e sem faixas etárias. Tendo como variação o período de um mês até seis meses para os aparecimentos do quadro após o evento estressor (OMS, 1993). Após a exposição ao evento estressor, começa uma série de sintomas específicos biopsicossociais, o que classifica o TEPT nos transtornos de ansiedade e sub classifica-o em agudo devido a duração de um a três meses, crônico quando a duração for superior a três meses e tardio ao início de seis meses após a exposição ao trauma. (APA, 2005)

Os fatores de riscos são divididos em: fatores pré-traumáticos, que podem ser, psicológicos, os quais incluem problemas emocionais na infância até os 6 anos de idade e transtornos mentais prévios; ambientais, que abrengem status sócio econômico mais baixo, grau de instrução inferior, exposição anterior ao trauma, adversidade na infância, características culturais e história psiquiátrica familiar; e genéticos e fisiológicos, incluem gênero feminino e idade mais jovem no momento da exposição ao trauma (SOUTO et al, 2012). Os fatores peritraumáticos limitam-se a ambientais, os quais incluem gravidade do trauma, ameaça percebida à vida, lesão pessoal, violência interpessoal e para pessoal militar testemunhar atrocidades. (SOUTO et al, 2012). Já os fatores pós-traumáticos podem ser: psicológicos, que abarcam avaliações negativas, estratégias de enfrentamento inapropriadas e desenvolvimento de transtorno de estresse agudo; ou ambientais, que incluem exposição a lembranças desagradáveis, eventos de vida adversos, perdas financeiras ou outras perdas relacionadas ao trauma. (SOUTO et al, 2012). Em relação às bases biológicas, o transtorno é visto como uma falha do organismo em se recuperar de eventos marcantes. Entre os hormônios importantes em nosso organismo, destaca-se o cortisol, cuja atividade pode interferir nos neurotransmissores como um mediador de resposta ao estresse, ou seja, quando o nível do cortisol está elevado nos sentimos mais fortes, motivados diminuindo o sentimento de cansaço e confuso. O cortisol se apresenta em quantidade reduzida em paciente com TEPT, por isso, existe a possibilidade de prever o desenvolvimento do transtorno no período imediatamente após o evento estressor. (GRAEFF, 2003). 
INVESTIGAÇÃO SOBRE SINTOMAS DE TRANSTORNO DE ESTRESSE PÓS-TRAUMÁTICO EM POLICIAIS:

UM ESTUDO A PARTIR DO GRUPO DE OPERAÇÕES ESPECIAIS (GOE) DE CACOAL-RO

Após a exposição ao evento traumático, o indivíduo pode ter recordações desta ocasião por alguns dias, o que é considerado normal. O quadro necessita de atenção caso, após meses ou anos, essas recordações retornem e esses sintomas se manifestem através de revivescência do trauma, em outras palavras, pensamentos recorrentes, pesadelos e flashback que remetem à lembrança do trauma; esquiva e isolamento social, evita ambientes, contatos e situações que possam Ihe trazer lembranças do trauma; e hiperexcitabilidade psíquica e psicomotora, batimentos cardíacos acelerados, insônia, sonolência, dificuldade em concentrar-se, irritabilidade, hipervigilância (DSM-5, 2014). Dentro os sintomas existentes para caracterizar o Transtorno de Estresse Pós-Traumático, podemos citar o sofrimento psicológico intenso ou reatividade fisiológica quando o indivíduo é exposto a eventos semelhantes ou que simbolizam algum aspecto do evento traumático, podendo ser como fator desencadeante uma sensação física para aqueles com quadros altamente somáticos. Alterações negativas persistentes nas cognições ou no humor podem provocar no indivíduo irritabilidade e comportamento imprudente ou autodestrutivo, como direção perigosa, uso excessivo de álcool e drogas, ou comportamento automutilante ou suicida. (DSM-5, 2014). Os sintomas, sua predominância e sua duração podem variar com o tempo. Aproximadamente, em metade dos casos com adultos a recuperação completa pode ser obtida em três meses, mas alguns indivíduos permanecem com os sintomas por meses e, às vezes, por mais de cinquenta anos. Tanto as recordações quanto novos eventos traumáticos podem levar a recorrência e a intensificação dos sintomas. Em indivíduos mais velhos pode haver deterioração da saúde e isolamento social. (DSM-5, 2014)

O TEPT pode vir associado a outros tipos de transtornos tais como; depressão, outros transtornos de ansiedade e o uso excessivo de substâncias psicoativas. (MCFARLANE, 1996). Os indivíduos com TEPT quanto mais rápido forem tratados, menor é o risco de comorbidades, que podem causar a potencialização do sofrimento e o aparecimento das consequências (MCFARLANE, 1996). A existência da comorbidade entre o TEPT e ADAD (abuso e dependência de álcool e drogas) tem um aumento significante no sexo masculino (KESSLER, 1995), (MCFARLEANE, 1993), mas ainda é necessário um enfoque maior na compreensão desse fenômeno para a realização de uma intervenção eficaz. Diante da polêmica existente entre ADAD e TEPT, não se sabe exatamente os fatores causadores da comorbidade. Dentro dessa causalidade, segundo Coffey, (2007), a diminuição dos sintomas de TEPT causaria concomitantemente a diminuição ou melhora do quadro de ADAD.

Devido à atividade executada com alta exposição às situações marcantes, esses profissionais das forças policiais podem desenvolver este transtorno (CÂMARA FILHO, 1999). O evento traumático é persistentemente revivido em recordações e sonhos aflitivos, e a pessoa pode agir ou sentir como se o evento traumático estivesse ocorrendo novamente (flashback), podendo esquivar-se na presença de estímulos relacionados ao trauma e ainda poderá desencadear 
sintomas persistentes de excitabilidade aumentada. Um fenômeno frequente e, aparentemente, paradoxal em várias destas pessoas é a reexposição compulsiva a novos eventos potencialmente traumáticos semelhantes a situação vivida no trauma (DSM-5, 2013). Por mais que prevaleça um alto índice do TEPT em relação a população, nem todos podem desencadear o TEPT ao se defrontar com um evento traumático, havendo uma necessidade de uma avaliação clínica das reações após a vivencia, podendo assim realizar uma intervenção terapêutica específica para cada quadro e elaborar formas de prevenção para aqueles que estão mais vulneráveis a psicopatologia (TAYLOR, 2006).

\subsection{Incidência do TEPT no Grupo de Operações Especiais}

Crank e Caldero (1991) apontam em seus estudos que atividades laborais praticadas pelos oficiais de polícia, como papeis burocráticos, seguido das exigências de tarefas e da violência ou perigo são atividades que mais causam estresse. Para Câmara Filho, (1999) o trabalho desenvolvido pelos Policiais Militares é considerado de grande risco para o desenvolvimento do Transtorno de Estresse Pós-Traumático devido a exposição a eventos estressores, podendo ser associado a outros transtornos mentais propiciando um desequilíbrio psicossocial e incapacidade de realização das atividades cotidianas. Esse autor utilizou o método de diátese-estresse para a Identificação do TEPT em relação as diferenças individuais pré-traumáticos e inespecíficos que dependeriam de outros fatores cujo a prevenção, identificação precoce e o tratamento do transtorno se dava a partir da integração dos elementos diátese biológica, genética e psicológica. Câmara Filho (1999) apresentou em sua dissertação um estudo prospectivo do Transtorno de Estresse Pós-Traumático em Policiais Militares com o objetivo de avaliar a incidência do quadro clínico em policiais que foram submetidos a eventos de traumas físicos por um período de um ano que, posteriormente, apresentaram fatores de risco. Os resultados, no primeiro mês, foram de aproximadamente $41,5 \%$ de incidência do Transtorno de Estresse Pós-Traumático e a prevalência de $14,6 \%$ em um ano, tendo como principal fator preditor a avaliação subjetiva de ameaça à vida para o desenvolvimento de TEPT agudo e crônico. Entretanto, segundo Câmara Filho (1999), não necessariamente todos os indivíduos expostos a eventos traumáticos desenvolvem o transtorno.

Uma pesquisa realizada com profissionais durante uma ajuda humanitária no Haiti, após o trágico terremoto de 2010, com mais de 300 vítimas fatais, trouxe um resultado com prevalência de TEPT nos trabalhadores que ajudaram no resgate. Este resultado equivale a uma variável de $19,8 \%$ a $42 \%$, nos quais os fatores de risco presentes foram: experiência prévia de trabalho em catástrofes, histórico de experiência de eventos traumáticos, baixo suporte social, sexo feminino, baixa idade, histórico de ansiedade e de depressão prévio ao evento, intervalo do tempo entre a última experiência estressante e a atual e tempo de exposição ao evento estressor. (SIMON, 2013) 
INVESTIGAÇÃO SOBRE SINTOMAS DE TRANSTORNO DE ESTRESSE PÓS-TRAUMÁTICO EM POLICIAIS:

UM ESTUDO A PARTIR DO GRUPO DE OPERAÇÕES ESPECIAIS (GOE) DE CACOAL-RO

O Governo do Estado de Santa Catarina desenvolveu o Programa de Gerenciamento de Estresse Profissional e Pós-traumático (PROGESP) com o objetivo de prevenir e promover o tratamento do Transtorno de Estresse Pós-Traumático nos policiais, considerados indivíduos vulneráveis aos eventos estressores e desencadeadores do transtorno. Em 2013, o Governo de SC deslocou para cada região um psicólogo para fazer parte da equipe PROGESP. Desta forma, o PROGESP realiza ações diretivas na educação dos policiais militares para o autogerenciamento de estresse na prevenção do TEPT, ao serem submetidos a ocorrências de incidente crítico como (ocorrência que tenha causado impacto) emocional nos policiais; morte ou lesão grave; vítimas fatais ou poli-traumatizadas; confronto com a utilização de armas de fogo com o resultado de morte ou ferimento de cidadão civil, etc. O comandante responsável aciona o PROGESP através do preenchimento do formulário Registro de Incidente Crítico (RIC), mobilizando a equipe do programa.

Carvalho, (2009) apresentou sua tese de Mestrado em Psicologia Clínica e de Saúde, Stress e Sintomas de Estresse Pós-Traumático - a PSP nas Missões Internacionais, com base em três modalidades de transtornos de estresse decorrentes de eventos traumáticos: estresse tardio, estresse agudo ou reação aguda ao estresse e o estresse pós-traumático ou incidental propriamente dito. Afirmando que esse conjunto de reações tem como base a relação do indivíduo com o ambiente quando este tem a percepção que está sendo ameaçado ou afrontado, mediante sua funcionalidade, causando um desconforto físico e mental, alterações no comportamento pessoal, familiar e social.

o fato de o indivíduo estar em contato direto ou próximo ao evento traumático a probabilidade de desenvolver o transtorno é maior, a falta de experiência do profissional para lidar com situações estressantes, o momento pessoal que o profissional está passando no momento de sua vida também influenciam no desenvolvimento do transtorno, assuntos particulares mal resolvidos, o nível de ansiedade elevando são algumas das variáveis de grande importância para o desenvolvimento de TEPT (MITCHELL; BRAY, 1990).

\section{Método}

\subsection{Características da População}

A pesquisa foi realizada na instituição do $4^{\circ}$ Batalhão de Polícia em Cacoal. O grupo surgiu em 2001, na cidade de Cacoal, especializado em atuar em ocorrências atípicas, como sequestros, assalto a banco, rebeliões em presídios, reintegração de posse, entre outros. São policiais altamente especializados e qualificados através de treinamentos expostos a situações que podem ocorrer, submetidos a fatores de estresse, de alta complexidade e alto risco. 


\subsection{Amostra}

O Grupo de Operações Especiais (GOE) de Cacoal- RO possui 12 integrantes. Deste total, 09 (nove) sujeitos foram escolhidos por meio de convite realizado no $4^{\circ}$ Batalhão de Polícia, com idades entre 28 a 45 anos, de ambos os sexos (masculino/feminino).

\subsection{Tipo de Pesquisa}

Por ser uma pesquisa de campo, tem como características fundamentais a investigações, na qual, além da pesquisa bibliográfica ou documental, se realiza coleta de dados em tempo real, junto ao indivíduo (FONSECA, 2002). Essa pesquisa terá como método a abordagem quantitativa e qualitativa, e será um estudo de levantamento descritivo. O uso da abordagem qualitativa está relacionado a investigação do fenômeno a ser pesquisado e as suas relações com o meio, dando ênfase ao contato direto. (GIL, 1999). Por ser quantitativa, as características dos participantes são descritas e técnicas padronizadas são utilizadas para coleta de dados como a entrevista por meio do "questionário". As pesquisas descritivas, segundo Gil (1999), têm como finalidade principal a descrição das características de uma determinada população ou fenômeno ou estabelecimento de relações entre as variáveis utilizando formas padronizadas para coleta de dados.

\subsection{Procedimento e Materiais da pesquisa}

O projeto, por meio da carta de anuência para o Comitê de Ética e Pesquisa (CEP) da Faculdade, através da Plataforma Brasil, obteve a aprovação ética dos procedimentos metodológicos para coleta de dados.

Inicialmente apresentou-se o projeto de pesquisa para o $4^{\circ}$ Batalhão de Polícia de Cacoal, com o intuito de obter a carta de anuência para desenvolvimento da pesquisa. A coleta foi realizada no próprio quartel, utilizando a sala cedida pela própria instituição, que possui estrutura propícia a aplicação do questionário sem inferências visuais e auditivas, bem como o sigilo dos sujeitos avaliados. Todos os policias da instituição foram comunicados sobre a pesquisa e sua importância para os profissionais de sua área. Assim, os policiais que tiverem interesse em participar da pesquisa assinaram o TCLE, compreendendo todos os objetivos e procedimentos da pesquisa.

Após o resultado dos questionários, os participantes receberam uma devolutiva individual e encaminhados para uma avalição psicológica, o que não foi necessário. Por se tratar de uma pesquisa com seres humanos, o projeto atendeu todas diretrizes e normas da resolução $\mathrm{N}^{\circ} 466$, de 12 de dezembro de 2012, com fundamentos éticos descritos. 
INVESTIGAÇÃO SOBRE SINTOMAS DE TRANSTORNO DE ESTRESSE PÓS-TRAUMÁTICO EM POLICIAIS:

UM ESTUDO A PARTIR DO GRUPO DE OPERAÇÕES ESPECIAIS (GOE) DE CACOAL-RO

\subsection{Técnicas de coleta de dados}

Na etapa quantitativa, a coleta de dados foi por meio de aplicação de um instrumento desenvolvido em 1993 Weathers, Litz, Huska e Keane, do National Center for PTSD (EUA, 1993) Post-Traumatic Stress Disorder ChecklistCivilian Version (PCL-C), para avaliar as consequências de eventos traumáticos. A PCL-C pode ser utilizada por diversos grupos etários e para diversos eventos traumáticos. É um instrumento de fácil aplicação e de curta duração, aproximadamente 10 minutos.

Em vários tipos de estudos o instrumento tem apresentado uma característica psicométricas satisfatórias em relação a validação e confiabilidade (BERGER, 2004). A característica da escala de PCL-C é composta por 17 itens, divididos em três dimensões, nas quais incluem, nos itens 1, 2, 3, 4 e 5, a reexperiência do trauma, a evitação nos itens $6,7,8,9,10,11$ e 12 e a hiperestimulação que abrange os itens 13, 14, 15, 16 e 17.

\section{Resultados}

Quanto ao perfil dos sujeitos (Tabela 1), as idades variam em 28 a 45 anos $(33,88)$, sendo que 04 (quatro) estão acima de 30 anos; o tempo de profissão está entre 6 a 24 anos (11,77). Quanto ao nível de escolaridade, a maioria cursou o ensino médio e seu o estado civil variou entre casados (maioria), viúvos e solteiros:

Tabela 1 - Perfil dos Sujeitos

\begin{tabular}{ccccc}
\hline Sujeitos & Idade & Tempo de Profissão & Escolaridade & Estado Civil \\
\hline $\mathbf{1}$ & 28 & 6 & Ensino Médio & Casado \\
\hline $\mathbf{2}$ & 28 & 6 & Ensino Médio & Viúvo \\
$\mathbf{3}$ & 29 & 6 & Superior & Casado \\
$\mathbf{4}$ & 30 & 9 & Ensino Médio & Viúvo \\
$\mathbf{5}$ & 31 & 9 & Superior & Solteiro \\
$\mathbf{6}$ & 34 & 14 & Ensino Médio & Casado \\
$\mathbf{7}$ & 36 & 14 & Ensino Médio & Casado \\
\hline $\mathbf{8}$ & 44 & 18 & Ensino Médio & Solteiro \\
$\mathbf{9}$ & 45 & 24 & Ensino Médio & Casado \\
\hline Fonte: Dados da pesquisa & & & \\
\hline
\end{tabular}

Na análise dos resultados do Post-Traumatic Stress Disorder Checklist Civilian Version (PCL-C), aplicado em 09 (nove) participantes, obtivemos uma prevalência de $100 \%$ do ponto de corte. Segundo Berger, Mendlowicz, Souza e Figueira, (1993), o escore maior ou igual a 50 pontos é considerado como provável caso de TEPT, porém, todos tiveram uma pontuação inferior a 50 pontos. No entanto há uma prevalência em 03 (três) participantes (33,33\%), que obtiveram a pontuação de 28-29 (alguns sintomas) e de 31-35 (moderada ou moderadamente alta severidade dos sintomas) em mais 03 (três) participantes (33,33\%) - (Tabela 2 e Gráfico 1): 
Tabela 2 - Nível de Sintomas e Diagnóstico

\begin{tabular}{ccl}
\hline Sujeito & Resultados dos Escores & Critérios preenchidos/ provável diagnóstico \\
\hline 1 & 31 & Moderada a moderadamente alta severidade dos sintomas \\
2 & 21 & Pouca ou Nenhuma Gravidade \\
3 & 21 & Pouca ou Nenhuma Gravidade \\
4 & 32 & Moderada a moderadamente alta severidade dos sintomas \\
5 & 23 & Pouca ou Nenhuma Gravidade \\
6 & 29 & Alguns Sintomas \\
7 & 35 & Moderada a moderadamente alta severidade dos sintomas \\
8 & 28 & Alguns Sintomas \\
9 & 29 & Alguns Sintomas \\
\hline Fonte: Dados da pesquisa & \\
\hline
\end{tabular}

Gráfico 1 - Nível de Sintomas e Diagnóstico

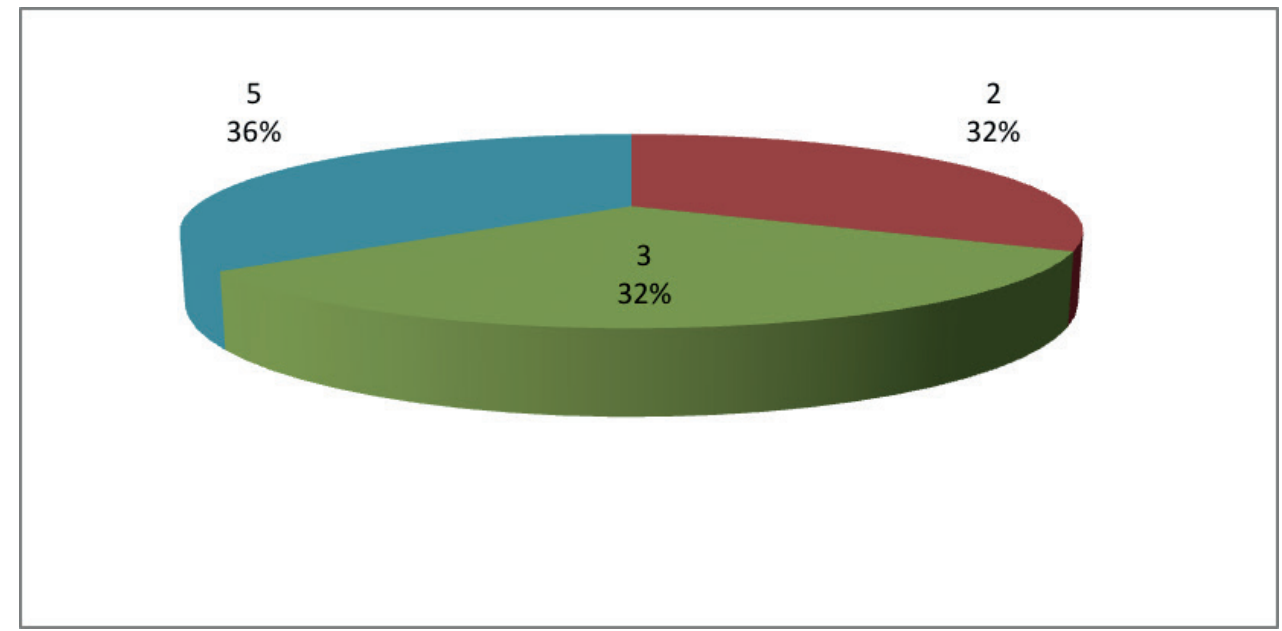

Fonte: Dados da pesquisa

A partir dos resultados obtidos consta-se, abaixo, os níveis de sintomas que se destacaram na análise da escala PCL.

Gráfico 1- Resposta Grupal- PCL nível “MÉDIO” dos sintomas.

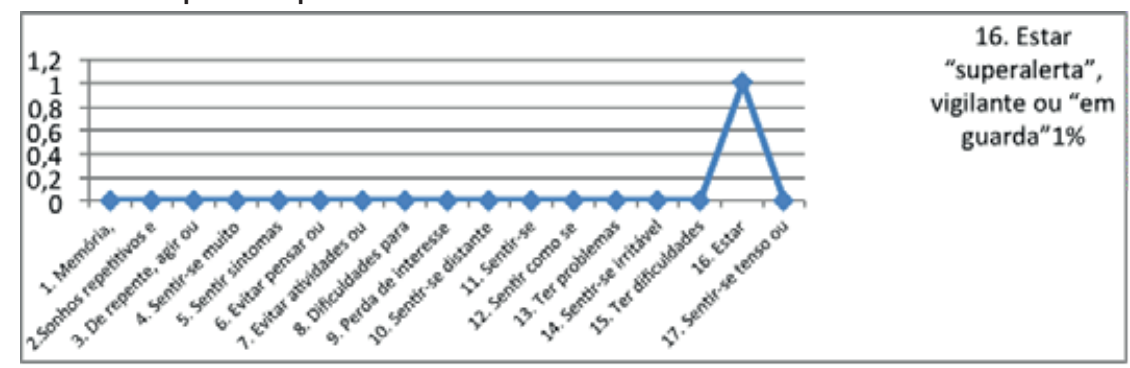


INVESTIGAÇÃO SOBRE SINTOMAS DE TRANSTORNO DE ESTRESSE PÓS-TRAUMÁTICO EM POLICIAIS:

UM ESTUDO A PARTIR DO GRUPO DE OPERAÇÕES ESPECIAIS (GOE) DE CACOAL-RO

O gráfico 01 apresenta nível "MÉDIO" dos sintomas relacionados a traumas, 04 (quatro) dos participantes destacam-se na dimensão dos sintomas de reexperiência do trauma, 06 (seis) na dimensão de evitação e 04 (quatro) na hiperestimulação.

Gráfico 2 - Resposta Grupal- PCL nível "BASTANTE" dos sintomas

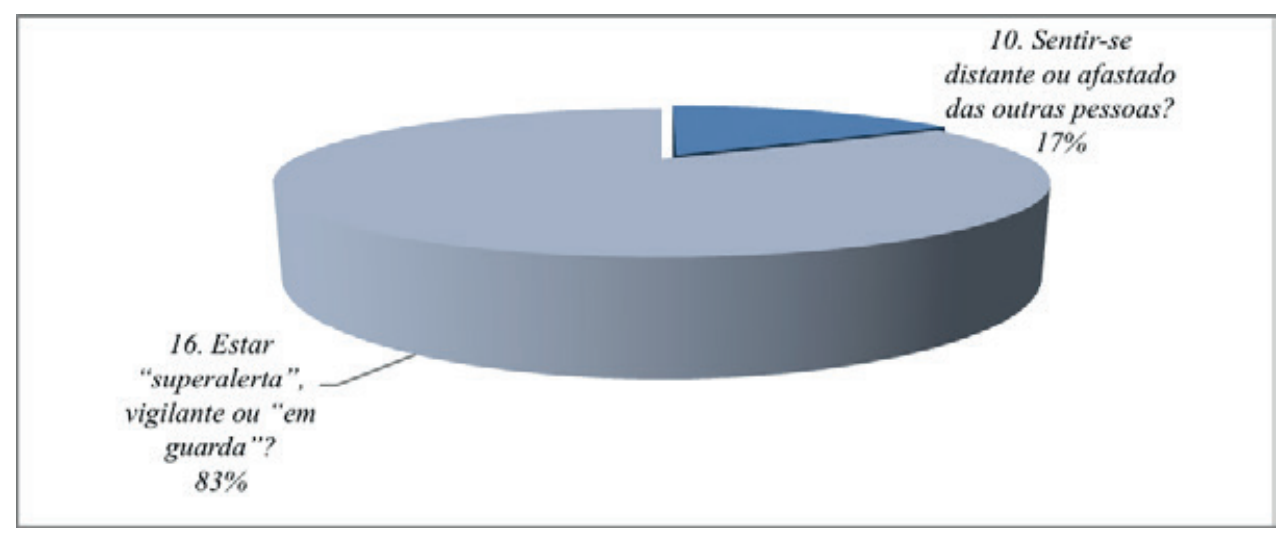

Fonte: Dados da pesquisa

O gráfico 02 apresenta o nível considerado "BASTANTE", nota-se que dos, 09 (nove) participantes, 05 (cinco) se sentem "BASTANTE", em estado de "superalerta", vigilante ou em guarda e 01 (um) sujeito se sente distante ou afastado de outras pessoas.

Gráfico 3 - Resposta Grupal- PCL nível “MUITO" dos sintomas

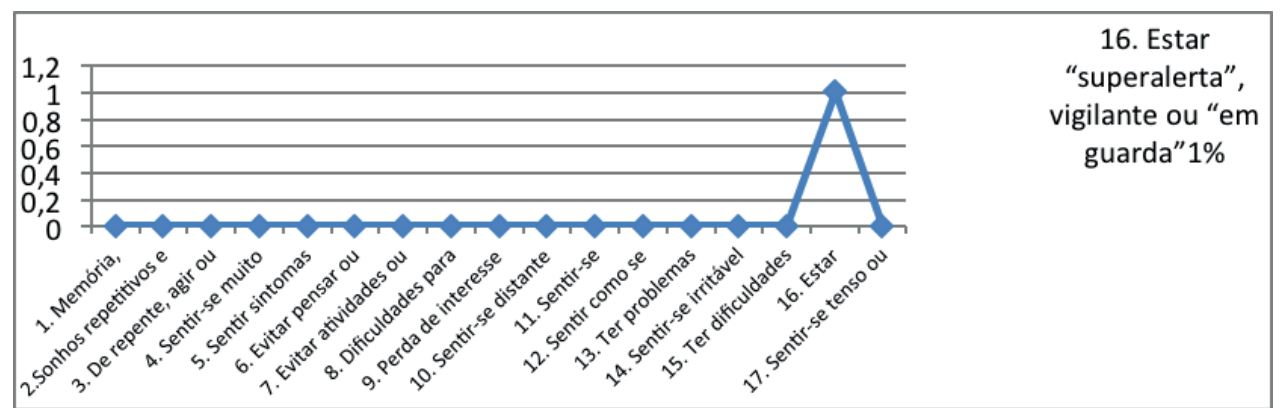

Fonte: Dados da pesquisa

O gráfico 03 apresenta o nível considerado "MUITO". Observou-se que 01 (um) sujeito se sente "MUITO" em estado de "superalerta", vigilante ou em guarda. Nos três gráficos apresentados há uma prevalência na terceira dimensão dos sintomas de hiperestimulação.

\section{Discussão}

Os resultados apontam um índice relevante na questão 16 do instrumento (Estar "superalerta", vigilante ou "em guarda"). No último mês,08 (oito) dos indivíduos disseram sentir-se incomodados, isso nos leva a um percentual de $88,9 \%$, um dado que remete à um olhar diferenciado e ao mesmo tempo pre- 
ocupante em questionar até que ponto esse "estar superalerta" não é prejudicial ao desempenho na corporação e até mesmo no convívio social e familiar.

O estado de "superalerta ou hipervigilância" é um estado tenso, ter uma percepção ampla de seus arredores. A hipervigilância está presente em alguns momentos de nossa vida de forma moderada, o que é considerado normal em certas situações no cotidiano, contudo, viver em estado de alerta o tempo todo causa prejuízo ao ser humano, principalmente aos policiais que são aqueles que se colocam em risco para manter a segurança dos demais. Este estado pode perturbar o sono, causar ansiedade ou nervosismo, esquiva, deixando o indivíduo propenso a ficar irritável e explosivo., além de ser extremamente cansativo, A hipervigilância é um dos sintomas presentes no TEPT (FIGUEIRA; MEDLOWICZ, 2003), deve-se, portanto, fazer uma investigação com o intuito de saber até que ponto esse estado superalerta está atuando como um fator de prejuízo significativo na vida dos sujeitos participantes desta pesquisa.

Pela quantidade de sujeitos que fazem parte do GOE (Grupo de Operações Especiais), 02 (dois) sujeitos sentindo-se distantes ou afastados das outras pessoas é um número relevante. Posto que a interação entre os membros da corporação é essencial para o desenvolvimento e execução das missões direcionadas, há uma necessidade de todos estarem em repleta comunicação e harmonia entre si. Campos (2004) diz que o apoio social pode contribuir para que a saúde se mantenha durante períodos de estresse, como um mediador proporcionando uma melhor aceitação diante de perdas ou problemas do cotidiano, afirmando que a integração entre eles é favorável para prevenção de doenças e em caso de serem acometidos por patologias o prognostico é mais eficaz. A interação e o apoio social estabelecem bem-estar psicológico relacionado ao nível de satisfação de saúde, autoestima e diminuição no sintoma da ansiedade, prevenindo um sofrimento psíquico, problemas relacionados ao sistema nervoso, dores crônicas e até mesmo o consumo exacerbado de substancias químicas (cigarro, álcool, drogas) entre outros problemas de saúde. (CAMPOS, 2004).

\section{Conclusão e considerações finais}

Embora o resultado de ocorrência de Transtorno de Estresse Pós-Traumático tenha sido negativo, esses dados promovem um alerta em relação a sintomatologia existente em alguns sujeitos participante. Pode-se reafirmar que alguns sintomas de TEPT estão presentes em grande parte dos participantes: os dados apresentados comprovam que houve um índice relevante na dimensão de reexperiência e hiperestimulação da escala $\mathrm{PCL}$, principalmente na dimensão de hiperestimulação, que remete um olhar atencioso em relação à saúde mental dos sujeitos participantes. A vulnerabilidade em relação a exposição no qual os policias estão sujeitos podem ser variáveis importantes na explicação de alguns sintomas de TEPT presentes.

Pode-se inferir que o indivíduo que mantem um contato direto ou indi- 
INVESTIGAÇÃO SOBRE SINTOMAS DE TRANSTORNO DE ESTRESSE PÓS-TRAUMÁTICO EM POLICIAIS:

UM ESTUDO A PARTIR DO GRUPO DE OPERAÇÕES ESPECIAIS (GOE) DE CACOAL-RO

reto com eventos traumáticos está propício a desenvolver o Transtorno de Estresse Pós-Traumático, bem como nível de ansiedade elevado também contribui para o desenvolvimento de TEPT (KNAPP; CAMINHA, 2003). Para lidar com essas situações estressantes são necessários manejos adaptativos. Os resultados apontam ainda que os policiais precisam cientificar-se sobre os danos que os sintomas do TEPT podem causar no contexto biopsicossocial. Programas direcionados à saúde dos policiais e conscientização sobre a vulnerabilidade são fundamentais para que a polícia possa desenvolver suas atividades e adequar-se aos fatores estressantes que muitas vezes são inevitáveis. O suporte social apontou-se como um recurso importante durante o processo de relações interpessoais seja eles no âmbito familiar, social ou institucional. Portanto é de grande relevância o apoio psicológico dentro da corporação, através de uma equipe multidisciplinar que promova a saúde física e mental, além da prevenção a possíveis transtornos mentais.

\section{Referências}

ASSOCIAÇÃO BRASILEIRA DE PSIQUIATRIA. Manual Diagnóstico e Estatística de Transtornos Mentais (DSM-V,2014) $5^{\mathrm{a}}$ ed pp 265,271-280

BARDIN, L. (1977). Análise de conteúdo. Lisboa: Edições 70.

BERGER, W., Mendlowicz, M. V., Souza, W. F., \& Figueira, I. (2004). Equivalência semântica da versão em português da Post-Traumatic Stress Disorder Checklist - Civilian VERSION (PCL-C) para rastreamento do transtorno de estresse pós-traumático. Revista de Psiquiatria do Rio Grande do Sul, 26(2), 167-175. doi: 10.1590/S010181082004000200006

BRINGHENTI, Luft e Oliveira (2010) Transtorno do estresse pós-traumático em acidentes de trânsito: validação de escala. Psico-USF (Impr.) vol.15 no.2 Itatiba May/Aug. 2010. Disponível em: http://dx.doi. org/10.1590/S1413-82712010000200007 Acesso em 05/07/2016.

CÂMARA FILHO, JWS. Sougey EB. Estupro e transtorno de estresse pós-traumático: aspectos epidemiológicos e clínicos. J Bras Psiquiatr 1999; 48:547-53.

CÂMARA FILHO; JWS. Transtorno de estresse pós-traumático: características clínicas e sócio demográficas em policiais militares e suas famílias (dissertação). Recife: Universidade Federal de Pernambuco; 1999.

CASTRO, S.L.S. Focalizando o trauma sob as lentes da clínica com policiais militares. Tese (Doutorado) Departamento de Psicologia da Pontificia Universidade Católica, Rio de Janeiro, 2009.

COFFER, S.F. Schumacher, J.A.Brady, K.T. Cotton, B.D. - Changes in PTSD symptomatology during acute and protracted alcohol and cocaine abstinence. Drug Alcohol Depend 87: 241-248, 2007.

CRANK, J. P. \& Caldero, M. (1991). The production of occupational stress in medium sized police agencies. A survey of line officers in eight municipal departments. Journal of Criminal Justice, 19, 339-349.

FILHO, E. Sougy. Transtorno de estresse pós-traumático: Formulação diagnóstica e questões de comorbidade.; Revista Brasileira de Psiquiatria, 2001; 23 (4) : 221-8.Disponível em http://www.scielo.br/pdf/rbp/ v23n4/7170.pdf

FONSECA, e Deodoro. Terapia cognitivo comportamental em grupo para o transtorno de estresse póstraumático. Psychiatry on line Brazil , 2007 vol.12 n7

FIGUEIRA, e Mendlowicz. Diagnóstico do transtorno de estresse pós-traumático; Revista Brasileira Psiquiatra, 2003: 25 (supl I): 12-6. Disponível em www.scielo.br/pdf/rbp/v25s1/a04v25s1.pdf. Acesso em 05/07/2016 
FOA, e Cashman, L. Jaycox, L. \& Perry.K. (1997). The validation of a self-report measure of PTSD: The Posttraumatic Diagnostic Scale. Psychological Assessment, 9, 445-451. Disponível em http://www.ptsd.va.gov/ professional/assessment/adult-sr/pds.asp

GIL, A. C. (1999). Métodos e técnicas em pesquisa social. São Paulo: Atlas.

GRAEFF, F. G. (2003). Bases biológicas do transtorno de estresse pós-traumático. Revista Brasileira de Psiquiatria, 25(supl. 1), 21-24.

KAPCZINSK, e Margis. Transtorno de estresse pós-traumático: Critérios diagnósticos; Revista Brasileira de Psiquiatria, 2003; 25 (supl I) :3-7. Disponível em: http://www.scielo.br/pdf/\%0D/rbp/v25s1/a02v25s1.pdf.

KESSLER, RC. Sonnega A.Bromet E. Hughes M. Nelson CB Posttraumatic stress disorder in the national comorbidity survey. Arch Gen Psychiatry 1995;52:1048-60.

LIMA, E. P.\& Assunção, A. A. (2011). Prevalência e fatores associados ao Transtorno de Estresse Pós-Traumático (TEPT) em profissionais de emergência: uma revisão sistemática da literatura. Revista Brasileira de Epidemiologia, 14(2), 217-230. doi: 10.1590/S1415-790X2011000200004

LIMA, Assunção e Barreto Transtorno de Estresse Pós-Traumático (TEPT) em Bombeiros de Belo Horizonte, Brasil: Prevalência e Fatores Ocupacionais Associados Psic.: Teor. e Pesq., Brasília, Abr-Jun 2015, Vol. 31 n. 2, pp. 279-288. Disponível em: http://www.scielo.br/pdf/ptp/v31n2/0102-3772-ptp-31-02-0279.pdf Acesso em 07/07/2016

MARGEN, e Picon. Relações entre estressores, estresse e ansiedade; Revista de Psiquiatria do RS, $2013 ; 25$ (supl I): 65-74. Disponível em http://www.scielo.br/pdf/rprs/v25s1/a08v25s1

MITCHELL, Jefft; BRAY, Grady P. Emergency Services Stress. 1 st ed. New Jersey: A Simon \& Schuster Company, 1990.

MCFARLANE, AC.Weber DL. Clark CR. Abnormal stimulus processingin posttraumatic stress disorder. Biol Psychiatry 1993;34:311-20. Disponível em www.scielo.br/scielo.php?script=sci_ arttext\&pid=S1516-44462001000400009. Acesso em 07/07/2016

MC FARLANE, AC. Avoidance and intrusion in posttraumatic stress disorder. J Nerv Ment Dis 1992;180:439-45. Internacional de Doenças e Problemas Relacionados à Saúde -Manual de Instruções - CID-10. 10ª revisão. v. 1. $3^{a}$ ed. São Paulo: editora da USP. 1996.

ORGANIZAÇÃO MUNDIAL DA SAÚDE, (1993). Classificação de transtornos mentais e de comportamento da CID-10: descrições clínicas e diretrizes diagnósticas. Porto Alegre: Artmed.

PROGESP, Programa de Gerenciamento de Estresse Profissional e Pós-Traumático. Disponível em: www.pm.sc.gov. br/.../programa-de-gerenciamento-de-estresse-profissional-e-pos-trau... Acesso em 04/07/2016.

PSICOSITE, Transtornos relacionados por semelhança ou classificação, Obtido via internet: www.psicosite.com.br/tra/ ans/estrespos.htm, Acesso em 23.09.2015.

PAULO, 2013. Transtorno de estresse pós-traumático (TEPT) em. Bombeiros de Belo Horizonte. Obtido via internet http://www.scielo.br/pdf/ptp/v31n2/0102-3772-ptp-31-02-0279.pdf Psicologia: Teoria e Pesquisa Abr-Jun 2015, Vol. 31 n. 2, pp. 279-288.

RAPHAEL, and J.P.Wilson (Eds.) Psychological Debriefing- theory, practice and evidence. Cambridge: Cambridge University Press

RIBEIRO, Transtorno de estresse pós-traumático, obtido via internet: www.minhavida.com.br/saude/.../ transtorno-do-estresse-pos-traumatico. Acesso em 04/06/2016.

SANTOS, Transtorno de estresse pós-traumático, obtido via internet: E Ferreira-Santos - 2007 - books.google.com, Acesso em 04/06/2016.

SIMON, Vannini, Santos, Silva e Andreoli. Sintomas de estresse pós-traumático em profissionais durante ajuda humanitária no Haiti, após o terremoto de 2010. Ciênc. saúde coletiva vol.18 no.11 Rio de Janeiro Nov. 2013. Disponívelhttp: //www.scielo.br/scielo.php?script=sci_arttext\&pid=S1413$81232013001100008 \&$ lang $=p t$

TAYLOR, S. (2006). Clinican's guide to PTSD: A cognitive-behavioral approach. New York: Guilford Press. 
INVESTIGAÇÃO SOBRE SINTOMAS DE TRANSTORNO DE ESTRESSE PÓS-TRAUMÁTICO EM POLICIAIS:

UM ESTUDO A PARTIR DO GRUPO DE OPERAÇÕES ESPECIAIS (GOE) DE CACOAL-RO

UNIVERSIDADE FEDERAL DE SÃO PAULO, Psicoterapia ajuda no combate ao transtorno de estresse pós-traumático, obtido via internet www.psiquiatria.unifesp.br/sobre/noticias/exibir/?id=63, Acesso em 21.06.2016.

VANNINI e Andreoli. Transtorno de estresse pós-traumático em pacientes de unidade de terapia intensiva; Revista Brasileira Ter Intensiva, 2010; 22 (1) :77-84.Disponível em www.scielo.br/pdf/rbti/v22n1/a13v22n1

WEATHERS, F. W., Litz, B. T., Herman, D. S., Huska, J. A. \& Keane, T. M. (1993). The PTSD checklist (PCL): reliability, validity, and diagnostic utility. Obtido em 22 de junho de 2016 do World Wide Web: 